\title{
Research on Input-output Efficiency of Urban Industrial Land based on Super-SBM Model
}

\author{
Quanhua Qian ${ }^{1}$ and $\mathrm{Xu} \mathrm{Lu}{ }^{1 *}$ \\ ${ }^{1}$ Department of Architecture and Urban Planning, Shenyang Jianzhu University, Shenyang, Liaoning, 110168, China
}

\begin{abstract}
The industrial land efficiency of 14 prefecture-level cities in Liaoning Province in China was measured and calculated by using three categories of input indicators (land, fixed asset, employed person) and output indicators (output value, profit, pollutant) and by using Super-SBM model. The results showed that the most of industrial land efficiency of every prefecture-level city in Liaoning Province was completely coordinated, the difference in their efficiency values was larger, the variable coefficient of their efficiency was large, and they had obvious two polarization; besides, they showed the decrease progressively from east to west on the spatial distribution and the trend of reducing from periphery to inner.
\end{abstract}

\section{Introduction}

As the spatial carrier of urban economy, society and environment, the utilization efficiency of urban land directly relates to the urban and social economic development and its living environment [1]. The industrial land efficiency evaluation is the important basis of realizing sustainable development under the background of the times when China's economic development pattern transforms. Since China adopted the reform and opening-up policy, the industrial land resources had been deemed as the pilot resources of local government for promoting investment attraction and creating local industrial clusters. Industrial land resources were facilitated to achieve the purpose of promoting the competitiveness enhancement of local economy $[2,3]$. Plenty of industrial parks and high-new technical parks newly emerge and expand rapidly, and land suffers with extensive utilization; such land supply manner results in the loss of land utilization efficiency [4, $5]$.

Liaoning Province is the traditional industrial base of China. However at the end of last century, Liaoning Province suffered with a declined economic development. After China proposed the strategy of revitalizing old northeast industrial base, China combined Liaoning coastal economic belt, Shenyang economic zone and other important economical plates to enhance economic vitality, and vigorously develop the high and new technical industry to drive the traditional industry to upgrade [6]. In view of the high land utilization degree in Liaoning Province, and the condition that the land utilization strength and land use efficiency were in the nationwide lower level, those prefecture-level cities in Liaoning Province were selected to study the industrial land utilization efficiency of Liaoning Province, and the empirical analysis was conducted by using the panel data about land transaction and deal case and industrial land on the micro-level in 2011 2016, as well as the SuperSBM model in this paper.

\section{Research methods and indicator selection}

The frequent traditional analysis method for studying urban land utilization efficiency is the DEA analysis method; the disadvantage of the analysis method is the one that the relative efficiency of decision-making unit does not consider the slack variable of unit input element, and can not process the problems related to the undesired value output. Therefore Tone $\mathrm{K}$. proposed the SBM (Slack Based Measure) model [7]; this model can simultaneously measure the inefficient conditions from two perspectives, namely input and output; it can not only solve the slack variable and its related problems, but also incorporate directly the undesired value output into the output [8]. Therefore, the SBM model, when considering to add the undesired output, provides more detailed tools for the analysis of industrial land efficiency of every prefecture-level city in Liaoning Province.

In this paper, the land utilization measure refers to the intensive use of industrial land in Liaoning Province. Due to the one that DEA model building needs to be measured by the data of input and output, the output data can be further divided into the desired output and the undesired output. Therefore, this paper also refers to the previous references on evaluation indicator, data availability and output-input element quantity $[9,10]$. After removing the highly self-correlation indicators, the industrial land market transaction area, input of industrial fixed assets and employed person of industry are served as the input indicators, the total industrial output value of

${ }^{*}$ Corresponding author's e-mail: luxupku@163.com 
the industrial enterprises above designated size and the total profits of industrial enterprises above designated size are served as the desired output elements, and the emission amount of industrial sulfur dioxide, industrial wastewater and industrial smoke and dust are served as the undesired output indicators.

The data of employed person of industry to be selected is originated from Statistical Yearbook of Urban Construction in China, and the fixed asset investment of industrial enterprises above designated size and other data are originated from China City Statistical Yearbook. The added value of industry, industry $\mathrm{SO}_{2}$, industrial wastewater, smoke and dust emission amount and other data are originated from Liaoning Statistical Yearbook, certain environmental quality report and environment yearbook.

\section{Industrial development in Liaoning Province, China}

Every prefecture-level city in Liaoning Province was used as the empirical object to evaluate the efficiency of status-quo industrial land. Assuming the scale efficiency is variable, analyze the inefficiency degree of input and the inefficiency degree of output, determine the ideal value of input and output, and obtain the improved value of input and output. Finally, the trend and characteristics of the SBM efficiency value of every prefecture-level city in Liaoning Province were analyzed from the perspective of panel data so as to seek the causes of their related phenomenon.

There are complete industrial categories in Liaoning Province, and Liaoning Province has 14 prefecture-level cities, 17 county-level cities, 8 national-level high and new zones and 7 provincial-level high and new zones. The pilot industry category in every prefecture-level city is distinctive. For instance, the pilot industries of Shenyang High and New Zone is information technology industry, intelligent manufacturing industry and biological medicine industry; the pilot industry of Dalian is software and service outsourcing industry. Early in 2004, Liaoning established Liaoning Province High and New Technical Industrial Development Promotion Association, and later in 2017 prepared Development Planning of High and New Technical Industry Development Zone in Liaoning Province (2017 2020). However, unsound use effect of industrial land and other realistic conditions may occur in the process of rapid urban development.

\section{Status analysis on efficiency of urban industrial land use}

The land use efficiency score to be obtained on the basis of the SBM model can elaborate the problems and rules of industrial land efficiency of every prefecture-level city. In order to make the efficiency value clearer, the method of type division in the existing references is borrowed [11]; when the efficiency value is more than or equal to 1 , it is the complete coordination; namely the output of the economic society and environment of the prefecturelevel city is relatively at the best level; when, it is the highly efficiency rate; when, it is the medium efficiency rate; when, it is the low inefficiency rate; when, it is the severe inefficiency rate.

All in all, in the Super-SBM model, the industrial land efficiency of every prefecture-level city in Liaoning Province in 2016 is shown by Table 2 as follows. According to the average value and variable coefficient of SBM land efficiency, it can be seen that the land efficiency of most of prefecture-level cities was the complete coordination, and it was characterized as follows:

- The overall level of the land efficiency of the high and new industry in every prefecture-level city in Liaoning Province currently was in the complete coordination. The average value of every prefecture-level city in Liaoning Province was $1.28(\geqslant 1)$, and the efficiency of 12 cities was completely coordinated, which is $85.7 \%$ of the total number.

- The difference in the industrial land efficiency value between every prefecture-level cities is expanding. Among which, the efficiency value of Anshan was lowest and it was only 0.31 , it became almost the severe inefficiency rate. The efficacy value of Fuxin was maximum (2.08), following it was Tieling and Benxi, the efficacy value was declined sharply. Between the last two cities, the efficacy value of Anshan was low inefficacy $(0.31)$, the efficacy value of Jinzhou was medium efficacy (0.68). The large variable coefficient of efficacy certifies the conclusion.

- The industrial land efficacy of inland cities was generally higher than that of coastal cities. Among coastal cities, Dandong City ranked in the first half; Anshan and Chaoyang, both inland cities ranked respectively top 14 and 8 .

Table 2. Evaluation of industrial land efficacy of prefecture-level city in Liaoning Province in 2016

\begin{tabular}{lcclcc}
\hline City & Efficacy value & $\begin{array}{l}\text { Ranking } \\
\text { placement }\end{array}$ & City & Efficacy value & $\begin{array}{l}\text { Ranking } \\
\text { placement }\end{array}$ \\
\hline Fuxin City & 2.08 & 1 & Chaoyang City & 1.21 & 8 \\
Tieling City & 1.73 & 2 & Huludao City & 1.16 & 9 \\
Benxi City & 1.66 & 3 & Dalian City & 1.12 & 10 \\
Liaoyang City & 1.62 & 4 & Yingkou City & 1.08 & 11 \\
Dadong City & 1.42 & 5 & Panjin City & 1.03 & 12 \\
Shenyang City & 1.42 & 6 & Jinzhou City & 0.66 & 13 \\
\hline
\end{tabular}




\begin{tabular}{|c|c|c|c|c|c|}
\hline Fushun City & 1.36 & 7 & Anshan City & 0.31 & 14 \\
\hline Average value & 1.28 & & $\begin{array}{l}\text { Variable } \\
\text { coefficient }\end{array}$ & 0.34 & \\
\hline
\end{tabular}

\section{Evolution analysis on industrial land efficacy}

Sort out the industrial land efficacy value of every prefecture-level city in Liaoning City during the period from 2011 to 2016, make the land efficacy change diagram reflecting its evolution process (Fig. 1), and the average value and variation coefficient change diagram (Fig. 2) of industrial land efficacy value, as well as the land efficacy change diagram of prefecture-level city suffering with low average value (Fig. 3). From which, it can be seen that the annual average efficacy level showed a fluctuating growth tendency, and the variable coefficient of efficacy was high and low irregularly, and the coordinated and regional difference of industrial land was huge; therefore the dynamic change characteristics of industrial land efficiency of every prefecture-level city are summarized into:

- Every prefecture-level city, namely Tieling, Huludao, Shenyang and Chaoyang, was in the advantage area of complete coordination, signifying that these prefecture-level cities had made outstanding achievements in land, labor force and capital input;
Fuxin, Liaoyang, Panjin, Dandong, Benxi, Jinzhou and Yingkou were in the medium average efficacy area, signifying the improvement in the input-output proportion of these cities and the coordination of other aspects; Dalian, Fushun and Anshan were in the low average efficacy area, signifying the output of its environment and social economy was not coordinated to its corresponding input proportion.

- Certain cities varied in the complete coordination and the non-coordination on the aspect of industrial land efficacy. Jinzhou suffered the decline from complete coordination to low inefficacy. Fuxin and Tieling varied from the medium completely coordination to the advantage area of complete coordination.

- Few cities suffered with an intensive fluctuation in land efficacy evolution. The cities suffered with larger change in efficacy value were Anshan, Fushun and Tieling; among which, the variable coefficient of Tieling was maximally 0.52 , it reflects the absolute value of efficacy's discrete degree. Anshan was always in the fluctuating medium inefficacy since the year 2012, it was possibly caused by the insufficient input and output or the negative benefit of output.

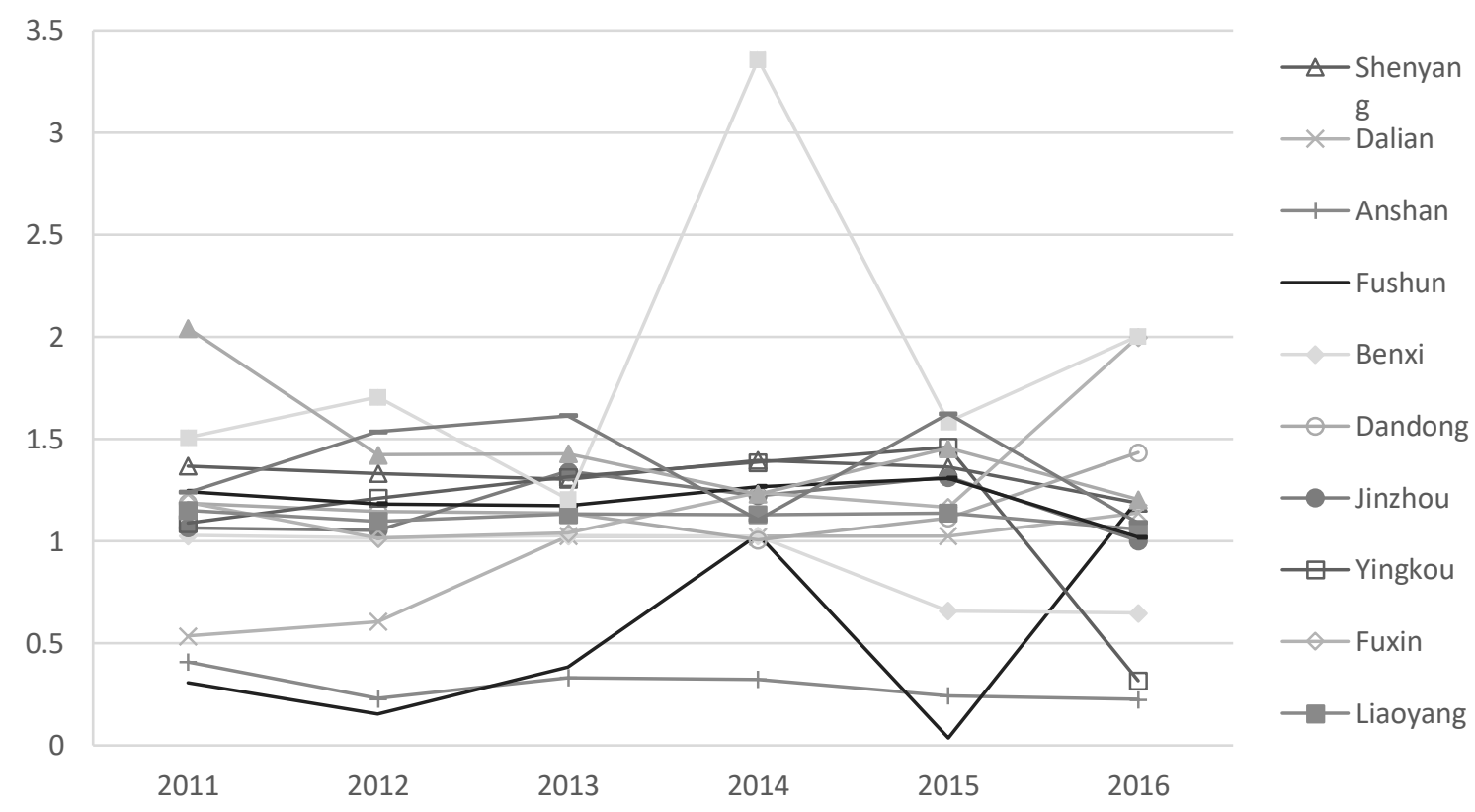

Fig. 1: Land use efficacy change diagram of cities in Liaoning, 2011-2016 


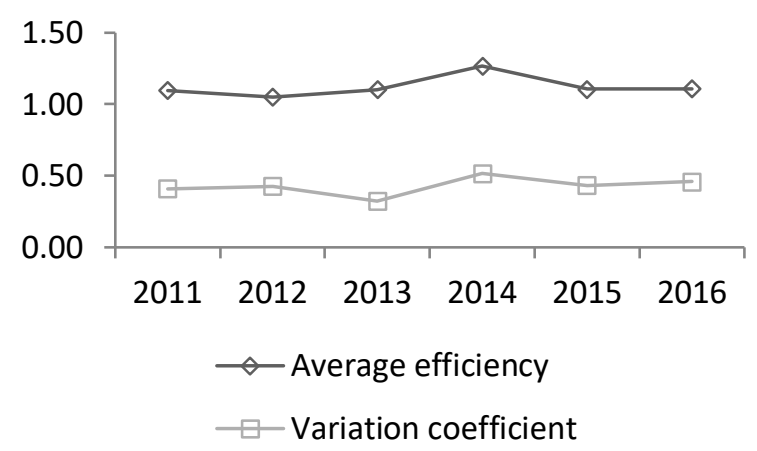

Fig. 2: Overall average value and variation coefficient

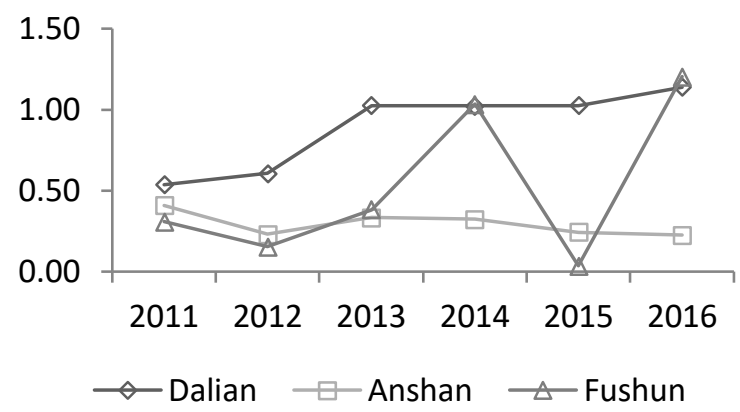

Fig. 3: Prefecture-level city suffering with low average value

\section{Conclusions}

The one to adopt the SBM model to research land efficacy is the major trend. In this paper in view of the insufficiency of existing results, new indicator variables were proposed to provide more accurate target to the data under research. By using the Super-SBM model taking the undesired output into consideration, analyzing the evaluation efficacy of industrial land of every prefecturelevel city in Liaoning in 2011 2016, the dynamic and static characteristics of land efficacy, as well as the projection performance on the production frontier level, we measured the relevant indicators, and obtained the following conclusions:

- The analysis on the static data and dynamic characteristics yielded the conclusion that most of high and new technical industry's land efficacy in Liaoning Province was of complete coordination. However the efficacy difference was larger, the maximum one of Fuxin was 7 times of the minimum one of Anshan; the variable coefficient of efficacy was large, and the polarized phenomenon was obvious. As for the spatially efficacy level, it showed the trend of reducing from east to west and from periphery to inner.

- By researching the result optimization of industrial land efficacy from the perspective of input and output inefficacy, it was found that there was generally the input redundancy, severe environmental pollution and other problems, and the unreasonable input and output proportion of every factor, which resulted in the ineffective use of resources; moreover the incoordination between undesired output and desired output resulted in the inefficacy of the output of prefecture-level city.

\section{Acknowledgments}

Authors are wishing to acknowledge financial support from the Energy Foundation.

\section{References}

1. David M, Eligius M T. (2000) A framework to study nearly optimal solutions of linear programming models developed for agricultural land use exploration. Ecological Modeling,131(5):65-77.

2. Li H, Zhou L. (2005) Political turnover and economy performance: the incentive role of personal control in China. Journal of Political Economics, 89: $1743-1762$.

3. Tao, R., Yuan, F., Cao, G.Z. (2007) Regional competition, land transfer and local financial effect: analysis of panel data of prefectural cities in China from 1999 to 2003. The Journal of World Economy, (10):15 - 27.

4. Wu, Q., Li Y. L. (2010) Fiscal decentralization, local government competition and land finance. Finance \& Trade Economics, (7): 51-59.

5. Luo, N.S., Peng, Y. (2016) The space-time analysis of urban industrial land use and the effects of the local government competition in China. China Land Sciences, (5): 62-70.

6. Zhou, R. (2015) Research on the development of high-tech industry in Liaoning. China Market, (4):33-34.

7. Tone, K. (2001) A slacks-based measure of efficiency in data envelopment analysis. European Journal of Operational Research, 130: 498 - 509.

8. Cheng, G. (2014). Data Envelopment Analysis: Methods and MaxDEA Software. Intellectual Property Publishing House, Beijing.

9. Li, H., Fang, K., Yang, W., Wang, D., Hong, X.X. (2013) Regional environmental efficiency evaluation in China: analysis based on the Super-SBM model with undesirable outputs. Mathematical and Computer Modelling, 58: 1018-1031.

10. Yang, Q.K., Duan, X.J., Ye, L., Zhang W. (2014) Efficiency evaluation of city land utilization in the Yangtze river delta using a SBM-Undesirable model. Resources Science, 36(4): 712 - 721.

11. Nie, L., Guo, Z.X., Peng, C. (2017). Construction land utilization efficiency based on SBMUndesirable and Meta-frontier model. Resources Science, 39: 836 - 845. 